\title{
Creencias en Salud Oral de Cuidadores de Pacientes Discapacitados y Adultos Mayores Institucionalizados de la Comuna de Quilaco, Región del BioBío
}

\author{
Oral Health Beliefs in Caregivers of Institutionalized Disabled \\ and Elderly Patients of the Quilaco Commune, BioBío Region
}

Camila Fuentes P.;; Carla Escobar I.; Patricia Muñoz M.* \& Nicolás Ernesto Ottone ${ }^{* *}$

FUENTES, P. C.; ESCOBAR, I. C.; MUÑOZ, M. P. \& OTTONE, N. E. Creencias en salud oral de cuidadores de pacientes discapacitados y adultos mayores institucionalizados de la comuna de Quilaco, Región del BioBío. Int. J. Odontostomat., 9(2):301-306, 2015.

RESUMEN: El envejecimiento demográfico acelerado de la población de Chile supone un aumento en la prevalencia no solo de enfermedades crónicas, sino también de personas con discapacidad, y necesidad de ser cuidadas en forma permanente. En este sentido, tomando como grupo de estudio a los cuidadores de los adultos mayores institucionalizados de la comuna de Quilaco, Región del Biobío, analizamos las creencias en salud oral que los cuidadores poseen al momento de llevar a cabo la atención de los adultos mayores que se encuentran a su cargo.

PALABRAS CLAVE: creencias en salud oral, cuidadores, adultos mayores institucionalizados.

\section{INTRODUCCIÓN}

Desde la segunda mitad del siglo XX nuestro país ha experimentado un proceso de envejecimiento demográfico acelerado y sin precedentes históricos. En la actualidad, una de cada diez personas pertenece al grupo adulto mayor y se espera que para el año 2025 esta proporción sea de uno por cada cinco (Instituto Nacional de Estadísticas, 2007; Nuñez \& Aspillaga, 2007). La mayor longevidad de la población implica no sólo una mayor prevalencia de enfermedades crónicas y degenerativas, sino también una mayor prevalencia de discapacidad, limitaciones funcionales y cognitivas, que afectan la independencia en el diario vivir de los adultos mayores (Departamento de Estudios y Superintendencia de Salud et al., 2008).

La Organización Mundial de la Salud (OMS) define discapacidad como "toda restricción o ausencia (debida a una deficiencia) de la capacidad de realizar una actividad en la forma o dentro del margen que se considera normal para un ser humano". Bajo este contexto, en el año 2004, el Fondo Nacional de la Discapacidad (Fonadis) llevó a cabo el "Primer Estudio
Nacional de la Discapacidad en Chile", el que estableció una prevalencia de discapacidad en nuestro país de un 12,9\% (Instituto Nacional de Estadísticas, 2004).

Toda persona que se encuentre en una situación de dependencia necesita de otro que lo cuide. Se establece en este caso, la figura del cuidador, el cual a su vez puede clasificarse como "formal" e "informal". El cuidador formal es aquella persona ajena al núcleo íntimo del individuo, que desarrolla la actividad de cuidar, de una manera profesional, siendo esta actividad remunerada. Por otra parte, se encuentra el cuidador informal, persona proveniente del círculo familiar o vecinal, que asume los cuidados de las personas no autónomas que están a su alrededor, desarrollando una función fundamental en nuestra sociedad (Burgos Garrido et al., 2008). Por lo tanto, de acuerdo a esta realidad social, resulta esencial medir las creencias en salud bucal de los cuidadores, ya sean estos formales o informales, para así poder determinar la prioridad que le asignan a la higiene bucal de los pacientes que cuidan (Garrido Urrutia et al., 2010).

\footnotetext{
* Profesionales independientes, Temuco, Chile.

"Facultad de Odontología, Universidad de La Frontera, Temuco, Chile.
} 
Dentro de los problemas de salud que aquejan a los adultos mayores, postrados o personas con discapacidad, las afecciones orales y dentales cobran especial importancia. Los problemas dentales no tratados causan dolor e incomodidad, interfiriendo con la capacidad de comer, dando como resultado una nutrición inadecuada que afecta a su bienestar general (Nuñez \& Quinteros, 2006).

El objetivo de nuestra investigación fue conocer las creencias en salud oral de los cuidadores de pacientes discapacitados dependientes y adultos mayores institucionalizados de la Comuna de Quilaco, y definir de este modo, el valor que los mismos otorgan a la salud oral en las personas que cuidan.

\section{MATERIAL Y MÉTODO}

Se realizó un estudio cualitativo, de tipo descriptivo, de corte transversal, para conocer las creencias en salud oral que poseen cuidadores de pacientes discapacitados dependientes y adultos mayores institucionalizados de la comuna de Quilaco, región del BioBío, Chile. En este estudio fueron incluidos cuidadores formales e informales de pacientes discapacitados dependientes o adultos mayores institucionalizados, pertenecientes al Programa de Atención Domiciliaria de la Posta de Quilaco y al Hogar de Ancianos Juan Pablo II de la comuna de Quilaco.

El universo total fue de 30 personas, 24 de las cuales pertenecían al Programa de Atención Domiciliaria y 6 de ellas eran cuidadores del Hogar de Ancianos Juan Pablo II. Del total de cuidadores no fueron encuestadas 6 personas, quienes no estuvieron de acuerdo con participar del estudio o no se encontraban en sus hogares en el momento de la visita, dejando un total de 24 personas encuestadas.

Para recolectar los datos se aplicó a los cuidadores la traducción del cuestionario Nursing Dental Coping Belifes Scale (DCBS) (Garrido Urrutia et al.). Los datos obtenidos a través de los instrumentos aplicados fueron transferidos, almacenados y analizados en planillas de datos del programa Microsoft $\circledast$ Office Excel 2003, para luego ser presentados en forma de tablas o gráficos según corresponda.

El cuestionario Nursing Dental Coping Belifes Scale (DCBS) evalúa a los cuidadores en 4 aspectos: locus de control interno, locus de control externo, autoeficacia y creencias para el cuidado de la salud oral, cada uno de los cuales incluye siete preguntas (ver Tabla I).

El locus de control interno se refiere a que la causa del éxito o fracaso es ubicado o percibido por la persona como algo interno a ella, como pueden ser sus habilidades y el esfuerzo que dedica a cada tarea e incluye preguntas como: creo que los dientes deberían durar toda la vida, creo que las caries pueden prevenirse, creo que usar la seda dental puede ayudar a prevenir las enfermedades de las encías, entre otras.

El locus de control externo se refiere a cuando la persona percibe que los resultados dependen de causas o factores externos, como pueden ser la suerte y el destino, se asume que el resultado no depende de la persona, sino de algo externo a ella, este ítem incluye preguntas como: sólo los dentistas pueden prevenir las caries y las enfermedades de las encías, si ambos padres tuvieron mala dentadura, el cepillado y el uso de seda dental no ayudará y creo que las prótesis dentales no tienen que ser removidas durante la noche, a menos que el paciente lo quiera, entre otras.

La dimensión autoeficacia incluye preguntas como: si les cepillo los dientes y uso la seda dental correctamente espero menos problemas dentales, creo que sé cómo se pueden tratar las diferentes enfermedades de la mucosa bucal y si me hubieran dado entrenamiento en el cuidado de la salud oral, sería capaz de realizar un mejor cuidado de la salud oral.

La dimensión creencias para la salud oral incluye preguntas como: creo que son los mismos pacientes quienes avisan cuando necesitan ayuda en el cuidado de su salud oral, creo que los productos fluorados son más adecuados para los niños y una vez que la enfermedad de las encías se ha iniciado, es casi imposible detenerla.

\section{RESULTADOS}

Caracterización de los cuidadores. En la caracterización de los cuidadores se observa que dentro de los cuidadores informales el mayor porcentaje corresponde a esposo(a), que en su mayoría son dueños(as) de casa y que poseen un nivel educacional básico incompleto (ver Tabla II). 
FUENTES, P. C.; ESCOBAR, I. C.; MUÑOZ, M. P. \& OTTONE, N. E. Creencias en salud oral de cuidadores de pacientes discapacitados y adultos mayores institucionalizados de la comuna de Quilaco, Región del BioBío. Int. J. Odontostomat., 9(2):301-306, 2015.

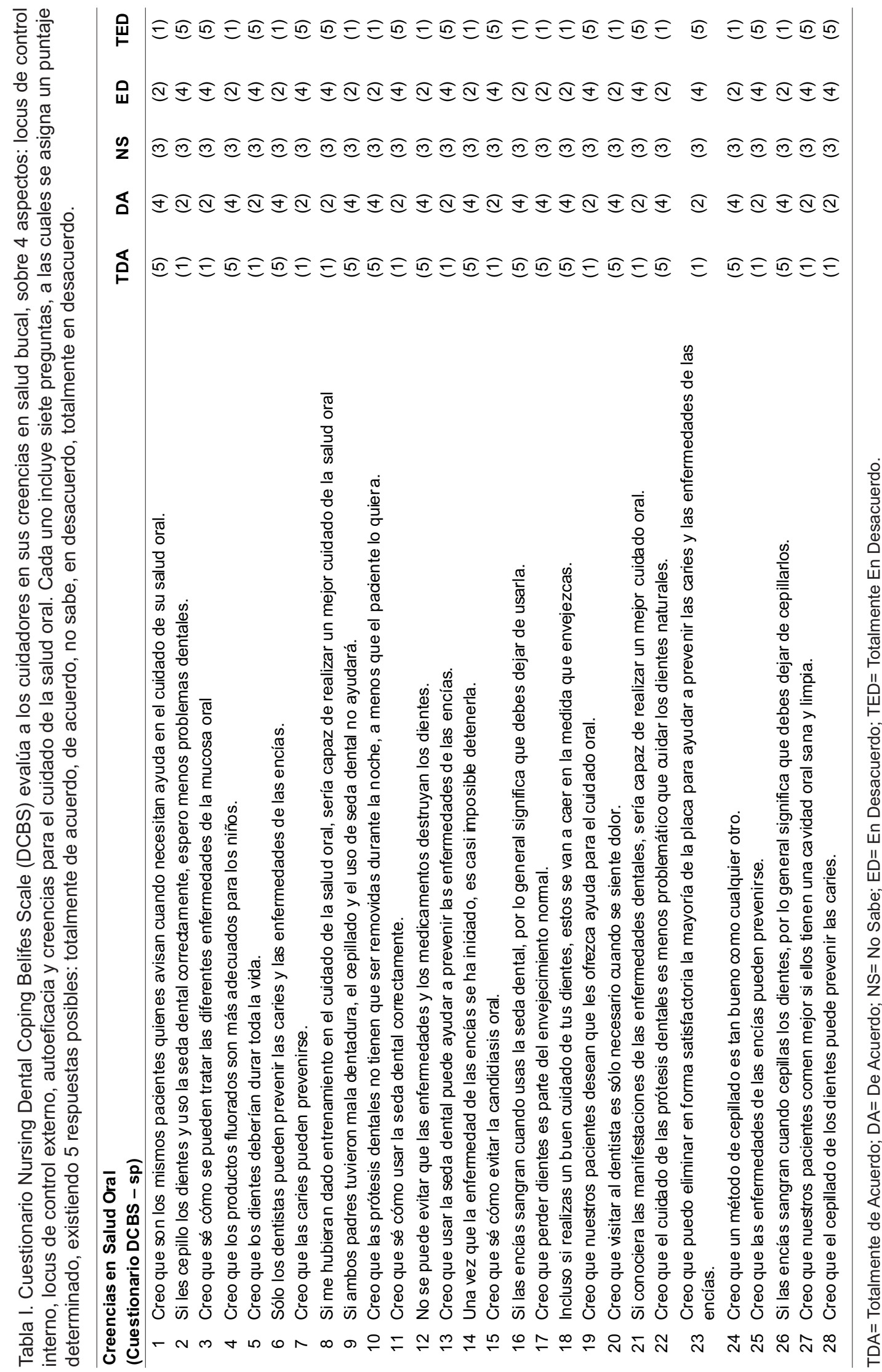


FUENTES, P. C.; ESCOBAR, I. C.; MUÑOZ, M. P. \& OTTONE, N. E. Creencias en salud oral de cuidadores de pacientes discapacitados y adultos mayores institucionalizados de la comuna de Quilaco, Región del BioBío. Int. J. Odontostomat., 9(2):301-306, 2015.

Tabla II. Caracterización de los cuidadores encuestados en la presente investigación, en referencia a su edad, formalidad, nivel de escolaridad, ocupación, entre otras cuestiones.

\begin{tabular}{lll}
\hline Edad promedio & 52,7 años & \\
Cuidadores formales & $29 \%$ & $35 \%$ \\
& Esposo(a) & $29 \%$ \\
& Hijo(a) & $12 \%$ \\
& Hermano(a) & $12 \%$ \\
Años promedio de desempeño & Madre & $12 \%$ \\
Ocupación actual & 4 años & \\
& Dueña de casa & $58 \%$ \\
& Trabajo tiempo completo & $25 \%$ \\
& Trabajo tiempo parcial & $13 \%$ \\
& Jubilado o pensionado & $4 \%$ \\
Nivel de escolaridad & Estudiante & $0 \%$ \\
& Otro & $0 \%$ \\
& Educación básica completa & $12 \%$ \\
¿Recibió capacitación en salud oral & Educación media incompleta & $8 \%$ \\
con anterioridad? & Educación media completa & $21 \%$ \\
\hline
\end{tabular}

Creencias en salud oral de los cuidadores. Cuidadores formales. En el ítem locus de control interno, estos cuidadores demostraron en un $88 \%$ poseer creencias positivas y en un $2 \%$ creencias negativas, al evaluar el ítem locus de control externo, se observó que en un $59 \%$ de los casos las creencias son positivas y en un $24 \%$ las creencias son negativas.

En el ítem autoeficacia, las creencias positivas de los cuidadores alcanzaron un $60 \%$ del total y las creencias negativas un 33\%. Asimismo, al evaluar el ítem creencias para la salud oral, estos cuidadores mostraron en un $67 \%$ de los casos poseer creencias positivas y sólo en un $19 \%$ creencias negativas.

En el caso de los cuidadores formales, vemos que en las cuatro dimensiones siempre la mayoría de las respuestas fueron positivas. Los mayores porcentajes de desconocimiento estuvieron en el caso de locus de control externo con un $24 \%$ y en creencias para la salud oral con un $19 \%$. Los resultados se pueden observar en la Figura 1.

Cuidadores informales. En el ítem locus de control interno, los cuidadores informales mostraron poseer en un $67 \%$ creencias positivas y en un $25 \%$ creencias negativas, mientras que en este caso, al evaluar el locus de control externo, se observa que las creencias positivas ascienden a un $45 \%$ y un $46 \%$ a creencias negativas.

En el ítem autoeficacia, los cuidadores informales presentaron un $50 \%$ de creencias positivas y un $42 \%$ de creencias negativas. Finalmente, en el ítem creencias para la salud oral, el porcentaje de creencias positivas fue de de $49 \%$ y el de creencias negativas de $38 \%$.

Para los cuidadores informales, la mayoría de las respuestas fueron positivas, sin embargo los porcentajes disminuyen en comparación con los cuidadores formales. Si observamos en el locus de control externo, al sumar el porcentaje de la respuesta "no sabe" con el porcentaje de respuestas negativas, el resultado de dicha suma supera el porcentaje de respuestas positivas. Esta situación se repite en la dimensión de creencias para la salud oral.

Los resultados correspondientes a los cuidadores informales se pueden observar en la Figura 2. 


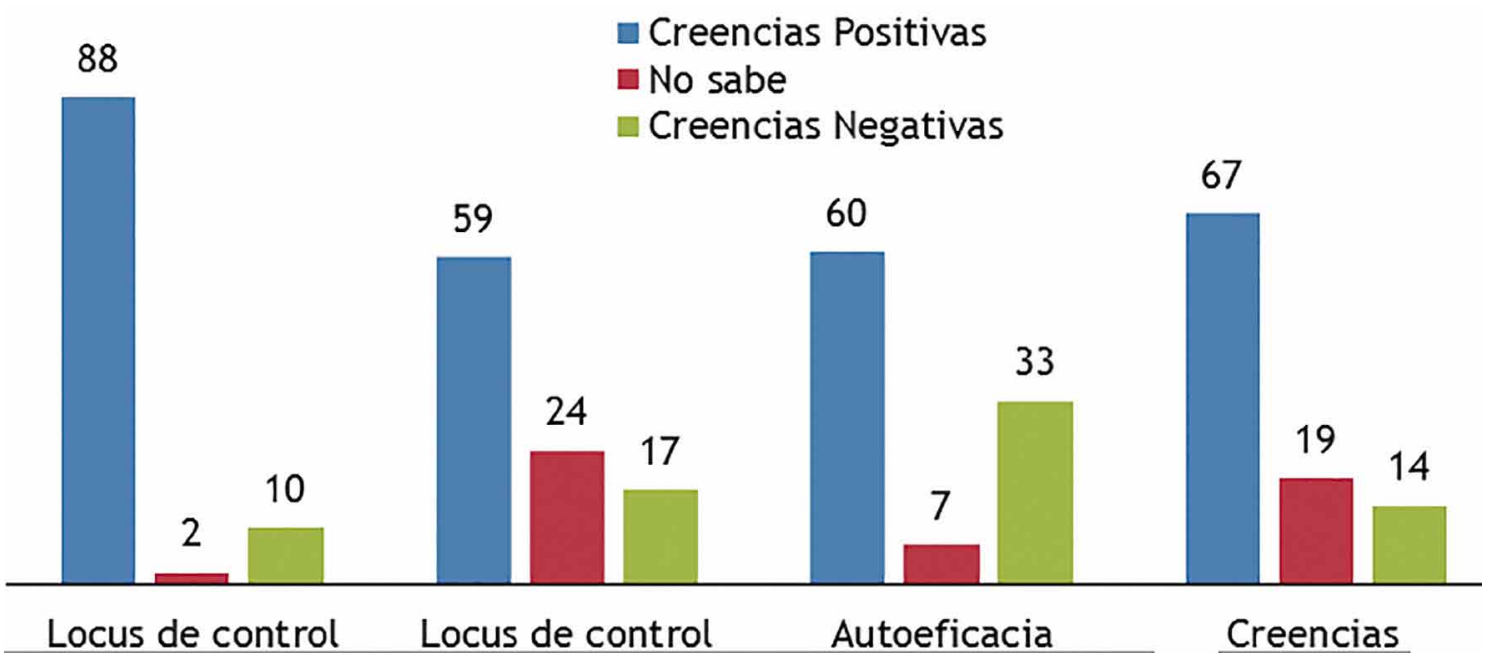

Fig. 1. Distribución de los resultados obtenidos de las encientas realizadas a los cuidadores formales en relación a las 4 dimensiones evaluadas con el Cuestionario Nursing Dental Coping Belifes Scale (DCBS).

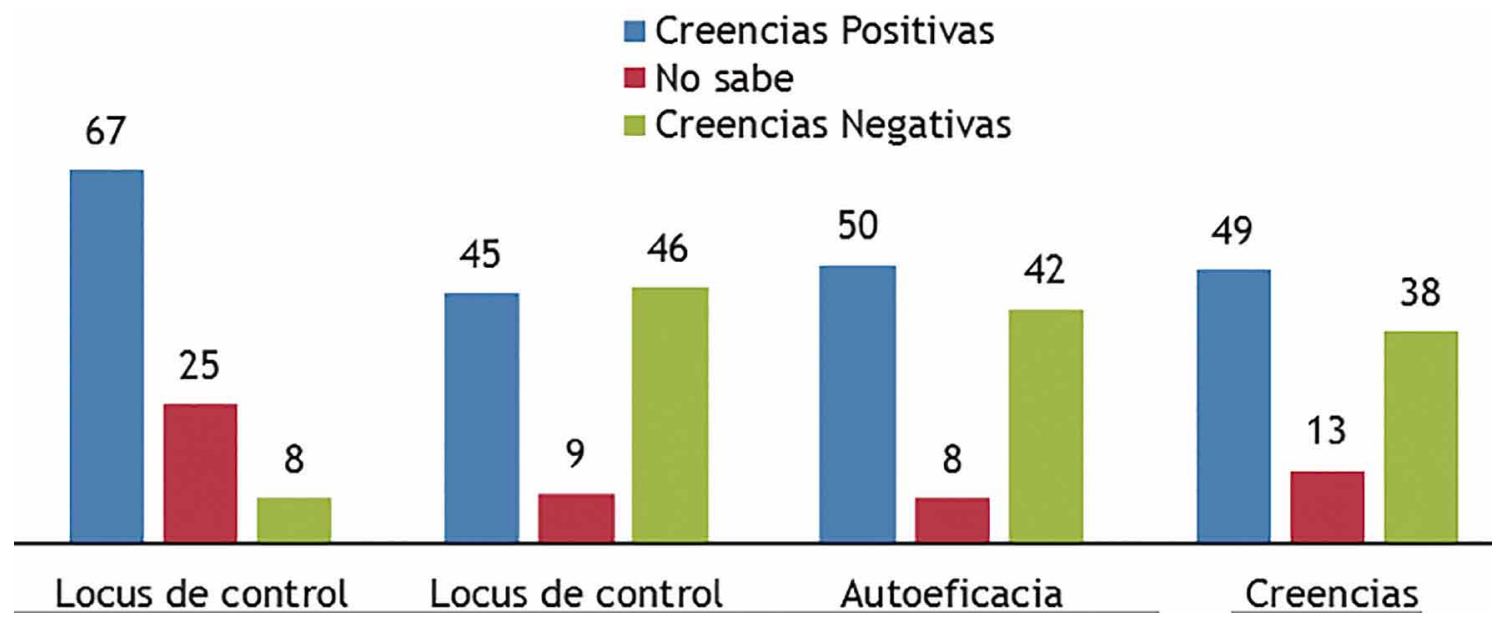

Fig. 2. Distribución de los resultados obtenidos de las encuestas realizadas a los cuidadores informales en relación a las 4 dimensiones evaluadas con el Cuestionario Nursing Dental Coping Belifes Scale (DCBS).

\section{DISCUSIÓN}

Estudios previos muestran que, en la mayoría de los casos, el cuidador corresponde a un hijo(a) (Nuñez \& Quinteros; Nuñez \& Aspillaga; Pizzarro et al., 2008), mientras que en nuestro estudio, el mayor porcentaje corresponde a esposo(a). Con respecto al estudio de Nuñez \& Aspillaga, se destaca como cuidador la hija, con un promedio de edad de 54,08 años, y con nivel educacional bajo.

En nuestra investigación, en la mayoría de los casos, el cuidador era mujer, dueña de casa, con un promedio de edad de 52,7 años. Estos resultados coinciden con los datos obtenidos en un estudio realizado en la comuna de Alto Hospicio en el año 2008 (Pizarro et al.).

En la comuna de Quilaco, la mayoría de los cuidadores lleva un promedio de 4 años realizando esta actividad, resultado similar al presentado por Pizarro et al., quien describe un rango de dedicación de entre 1 a 5 años. 
FUENTES, P. C.; ESCOBAR, I. C.; MUÑOZ, M. P. \& OTTONE, N. E. Creencias en salud oral de cuidadores de pacientes discapacitados y adultos mayores institucionalizados de la comuna de Quilaco, Región del BioBío. Int. J. Odontostomat., 9(2):301-306, 2015.

Los resultados coinciden con diversos estudios, al describir el bajo nivel de escolaridad que poseen los cuidadores, independientemente de si son formales o informales. En referencia a esto, un $46 \%$ de ellos poseen educación básica incompleta (Nuñez \& Quinteros; Pizarro et al; Luengo, 2010).

\section{CONCLUSIONES}

Al analizar los resultados que caracterizan las creencias para la salud, la mayoría de los casos son positivas en un porcentaje mayor al $40 \%$, siendo estas mayores en el grupo de cuidadores formales. La mayoría de los encuestados (75\%) reporta nunca haber recibido capacitación en el área del cuidado de la salud oral por parte del Equipo de Salud Municipal de Quilaco.

Este estudio permite visualizar que tanto en el caso de los cuidadores formales como informales existe un porcentaje no menor de desconocimiento sobre cuidado oral. Este desconocimiento se encuentra acentuado en el caso de los cuidadores informales.

A partir de los resultados obtenidos del cuestionario realizado, consideramos que es fundamental realizar una correcta capacitación de los cuidadores, tanto formales como informales, en el campo del cuidado de la salud bucal, la cual permitirá mejorar la atención que estas personas brindan a los adultos mayores de la comunidad de Quilaco, actuando como agentes de prevención en el desarrollo de las patologías orales que los pacientes, de acuerdo a su edad, puedan llegar a sufrir, y de este modo lograr un diagnóstico precoz y tratamiento a tiempo y adecuado de estas afecciones.

FUENTES, P. C.; ESCOBAR, I. C.; MUÑOZ, M. P. \& OTTONE, N. E. Oral health beliefs in caregivers of institutionalized disabled and elderly patients of the Quilaco commune, Biobío region. Int. J. Odontostomat., 9(2):301-306, 2015.

ABSTRACT: The rapid aging of the population of Chile will produce an increase not only in the prevalence of chronic diseases, but also of people with disabilities, and their need to be cared for permanently. In this sense, taking the caregivers of the institutionalized elderly population of the Quilaco commune, Biobío Region, as a study group, we analyzed the oral health beliefs that caregivers have at the time of undertaking the care of older adults.

KEY WORDS: oral health beliefs, caregivers, institutionalized elderly.

\section{REFERENCIAS BIBLIOGRÁFICAS}

Burgos Garrido, P.; Figueroa Rodríguez, V.; Fuentes Verdugo, M.; Quijada Hernández, I. \& Espinoza Lavoz, E. Caracterización y nivel de conocimiento del cuidador informal de usuarios con dependencia severa adscritos al centro de salud Violeta Parra - Chillán. Theor., 17(2):7-14, 2008.

Departamento de Estudios y Superintendencia de Salud - Departamento de Economía de la Salud, MINSAL - División de Planificación de Salud, MIDEPLAN. Dependencia de Adultos Mayores en Chile. Santiago de Chile, Gobierno de Chile, 2008. Disponible en: http://www.supersalud.gob.cl/documentacion/569/ articles-4471_recurso_1.pdf

Garrido Urrutia, C.; Espinoza Santander, I. \& Romo Ormazábal, F. Traducción al español y validación del cuestionario de creencias en salud bucal para cuidadores (DCBS-SP). Rev. Esp. Salud Pública, 84(4):407-14, 2010.

Instituto Nacional de Estadística (INE). Primer Estudio de la discapacidad e Informes Regionales. En: Gobierno de Chile, Ministerio de Planificación y Cooperación Fondo Nacional de la Discapacidad. Plan Nacional de Acción para la Integración Social de las Personas con Discapacidad 2004-2010. Santiago de Chile, Gobierno de Chile, 2004. Disponible en: http:// www.ministeriodesarrollosocial.gob.cl/btca/txtcompleto/ plan.integ.socpersdiscap2004-10.pdf

Instituto Nacional de Estadísticas (INE). Enfoque estadístico - Adulto Mayor. Santiago de Chile, Boletín informativo del Instituto Nacional de Estadísticas, 2007. Disponible en: http://www.ine.cl/ canales/sala_prensa/noticias/2007/septiembre/boletin/ ine_adulto_mayor.pdf

Luengo, C. E. Factores del cuidador familiar que influyen en el cumplimiento de los cuidados básicos del usuario postrado. Index Enferm., 19(1):14-8, 2010.

Nuñez, M. L. \& Quinteros, M. E. Diagnóstico en Salud Oral en pacientes postrados inscritos en el Consultorio Dr. José D. Astaburuaga, Talca. Talca, Escuela de Odontología, Universidad de Talca, 2006. Disponible en: http://di.utalca.cl/docs/pdf/ Memoria-2005-2006.pdf

Nuñez, M. L. \& Aspillaga, M. P. Diagnóstico de salud oral en personas mayores postradas inscritas en el Centro de Salud La Florida de la comuna de Talca. Talca, Escuela de Odontología, Universidad de Talca, 2007. Disponible en: http:// dspace.utalca.cl/handle/1950/4865

Pizarro, M. J.; Espinosa Arcuch, F.; Muñoz Uslar, L.; Peralta Oros, E. \& Laferte, M. Análisis descriptivo en cuidadores de personas postradas del consultorio Pedro Pulgar, comuna de Alto Hospicio. Rev. Soc. Med. VI Reg., 3(3):864-9, 2008.

Dirección para Correspondencia:

Camila Fuentes $P$.

Profesional Independiente

Temuco

CHILE

Email: caigfupi@gmail.com

Recibido : 18-08-2014

Aceptado: 22-05-2015 